\title{
Zeros of Dirichlet $L$-series on the critical line
}

by

\author{
Peter J. Bauer (Frankfurt/Main)
}

1. Introduction. In $1974, \mathrm{~N}$. Levinson showed that at least $1 / 3$ of the zeros of the Riemann $\zeta$-function are on the critical line ([19]). Today it is known (Conrey, [6]) that at least $40.77 \%$ of the zeros of $\zeta(s)$ are on the critical line and at least $40.1 \%$ are on the critical line and are simple. In [16] and [17], Hilano showed that Levinson's original result is also valid for Dirichlet $L$-series.

This paper is a shortened version of parts of the dissertation [3], the full details of which may be found at http://www.math.uni-frankfurt.de/ pbauer/diss.ps. We shall prove a mean value theorem for Dirichlet $L$-series and use this for proving some corollaries concerning the distribution of the zeros of $L$-series - amongst other results we improve the above mentioned bounds for Dirichlet $L$-series.

2. Notation, statement and comparison of results. Throughout this paper we will use the following notations. Let $T$ be sufficiently large, $\chi$ a primitive Dirichlet character and $q$ its modulus. Let

$$
\mathcal{L}:=\log \frac{q T}{2 \pi}
$$

and $A, B$ be complex constants satisfying $A \neq B, 0<|A|,|B|<\mathcal{A}$ for an arbitrary but fixed bound $\mathcal{A}$.

Using these notations our mean value theorem is

Theorem 1. Let $1 / 2 \leq c<1$ and $1 \leq q=o(\log T)$. Let $P_{1}$ and $P_{2}$ be polynomials with $P_{1}(0)=P_{2}(0)=0$. Choose a mollifier

$$
B_{y}\left(s, P_{j}, \chi\right):=\sum_{n \leq y} \frac{\chi(n) \mu(n)}{n^{s}} P_{j}\left(1-\frac{\log n}{\log y}\right) .
$$

2000 Mathematics Subject Classification: 11M26, 11M06. 
Define

$$
\begin{aligned}
\mathcal{M}:= & \frac{1}{i T} \int_{c+i}^{c+i T} L\left(s+\frac{A}{\mathcal{L}}, \chi\right) B_{y}\left(s, P_{1}, \chi\right) \\
& \times L\left(1-s-\frac{B}{\mathcal{L}}, \bar{\chi}\right) B_{y}\left(1-s, P_{2}, \bar{\chi}\right) d s .
\end{aligned}
$$

Then for every pair $\left(\theta_{1}, \theta_{2}\right)$ where $0<\theta_{1} \leq \theta_{2}<1 / 2$, there is a $\delta=$ $\delta\left(\theta_{1}, \theta_{2}\right)>0$ with the property that for every $\theta \in\left[\theta_{1}, \theta_{2}\right]$ and $y=T^{\theta}$,

$$
\begin{aligned}
\mathcal{M}= & \theta \cdot \frac{e^{B-A}-1}{B-A} \int_{0}^{1}\left(\frac{1}{\theta} P_{1}^{\prime}(t)-A P_{1}(t)\right)\left(\frac{1}{\theta} P_{2}^{\prime}(t)+B P_{2}(t)\right) d t \\
& +P_{1}(1) P_{2}(1)+O_{\mathcal{A}}\left(T^{-\delta}\right) .
\end{aligned}
$$

The implicit constant in the error term is independent of the parameters $\theta$, $A, B$, the character $\chi$ and its modulus $q$.

This theorem may be compared to results for $\zeta(s)([8],[6])$ or for Dirichlet series associated with holomorphic cusp forms ([13]).

Some applications of our theorem are as follows. Let

$N(T, \chi):=\#\{s: L(s, \chi)=0$, where $0<\sigma<1,0<t<T\}$,

$N_{0}(T, \chi):=\#\{s: L(s, \chi)=0$, where $\sigma=1 / 2,0<t<T\}$, $N_{0, \mathrm{~s}}(T, \chi):=\#\left\{s: L(s, \chi)=0, L^{\prime}(s, \chi) \neq 0\right.$, where $\left.\sigma=1 / 2,0<t<T\right\}$, and let

$$
\alpha(\chi)=\liminf _{T \rightarrow \infty} \frac{N_{0}(T, \chi)}{N(T, \chi)} \quad \text { and } \quad \alpha_{\mathbf{s}}(\chi)=\liminf _{T \rightarrow \infty} \frac{N_{0, \mathrm{~s}}(T, \chi)}{N(T, \chi)}
$$

denote the proportions of zeros and simple zeros on the critical line. Then we can show

Corollary 1. For any Dirichlet character $\chi$,

$$
\alpha(\chi)>0.365815 \text { and } \alpha_{\mathrm{s}}(\chi)>0.356269 \text {. }
$$

There is no need to restrict ourselves to primitive characters here because $L$-series to non-primitive characters share the same non-trivial zeros as the $L$-series to the corresponding primitive character.

Our bounds are weaker than the above mentioned bounds ([6]) known for $\zeta(s)=L\left(s, \chi_{0}\right)$ where $\chi_{0}$ is the (principal) character mod 1: $\alpha\left(\chi_{0}\right)>$ 0.4088 and $\alpha_{\mathrm{s}}\left(\chi_{0}\right)>0.4013$, slightly improved in [2] to $\alpha\left(\chi_{0}\right)>0.4089$ and $\alpha_{\mathrm{s}}\left(\chi_{0}\right)>0.4021$.

We obtain some further results concerning the multiplicity of the zeros and improve the results known for arbitrary Dirichlet characters ([16]).

The functional equation for $L(s, \chi)$ with a primitive character $\chi$ is

$$
H(s, \chi) L(s, \chi)=\varepsilon_{\chi} H(1-s, \bar{\chi}) L(1-s, \bar{\chi})
$$


where

$$
H(s, \chi)=\left(\frac{\pi}{q}\right)^{(s+a) / 2} \Gamma\left(\frac{s+a}{2}\right), \quad \text { where } \quad a= \begin{cases}0 & \text { if } \chi(-1)=1, \\ 1 & \text { if } \chi(-1)=-1,\end{cases}
$$

and

$$
\varepsilon_{\chi}=\frac{1}{i^{a} q^{1 / 2}} \sum_{\nu=1}^{q} \chi(\nu) e\left(\frac{\nu}{q}\right) .
$$

Here $\left|\varepsilon_{\chi}\right|=1$. Thus, if we choose a complex number $E_{\chi}$ satisfying $E_{\chi}^{2}=\bar{\varepsilon}_{\chi}$, we have $E_{\chi}^{-2}=\varepsilon_{\chi}$. Set

$$
\xi(s, \chi):=E_{\chi} H(s, \chi) L(s, \chi) .
$$

Then the functional equation can be restated as

$$
\xi(s, \chi)=\xi(1-s, \bar{\chi}) .
$$

If we define

$$
\begin{array}{r}
N^{(m)}(T, \chi):=\#\left\{s: \xi^{(m)}(s, \chi)=0, \text { where } 0<\sigma<1,0<t<T\right\}, \\
N_{0}^{(m)}(T, \chi):=\#\left\{s: \xi^{(m)}(s, \chi)=0, \text { where } \sigma=1 / 2,0<t<T\right\}, \\
N_{0, \mathrm{~s}}^{(m)}(T, \chi):=\#\left\{s: \xi^{(m)}(s, \chi)=0, \xi^{(m+1)}(s, \chi) \neq 0,\right. \\
\quad \text { where } \sigma=1 / 2,0<t<T\},
\end{array}
$$

as well as

$$
\alpha^{(m)}(\chi)=\liminf _{T \rightarrow \infty} \frac{N_{0}^{(m)}(T, \chi)}{N^{(m)}(T, \chi)} \quad \text { and } \quad \alpha_{\mathrm{s}}^{(m)}(\chi)=\liminf _{T \rightarrow \infty} \frac{N_{0, \mathrm{~s}}^{(m)}(T, \chi)}{N^{(m)}(T, \chi)},
$$

another consequence of Theorem 1 is

Corollary 2. For any Dirichlet character $\chi$, there are lower bounds

$$
\begin{array}{lll}
\alpha^{(1)}(\chi)>0.847212, & \alpha^{(2)}(\chi)>0.962736, & \alpha^{(3)}(\chi)>0.990523, \\
\alpha^{(4)}(\chi)>0.995581, & \alpha^{(5)}(\chi)>0.997573, & \alpha^{(6)}(\chi)>0.998093,
\end{array}
$$

and

$$
\begin{array}{lll}
\alpha_{\mathrm{s}}^{(1)}(\chi)>0.787784, & \alpha_{\mathrm{s}}^{(2)}(\chi)>0.931659, & \alpha_{\mathrm{s}}^{(3)}(\chi)>0.966755, \\
\alpha_{\mathrm{s}}^{(4)}(\chi)>0.979979, & \alpha_{\mathrm{s}}^{(5)}(\chi)>0.986488, & \alpha_{\mathrm{s}}^{(6)}(\chi)>0.990232 .
\end{array}
$$

Better bounds can be obtained for the special case of $\zeta(s)([3])$.

The bounds for simple zeros can, of course, be used to derive results concerning the multiplicity of the zeros. Let $\alpha \geq m(\chi)$ denote the proportion of zeros of $L(s, \chi)$ in the critical strip with multiplicity $\geq m$, and let $\alpha_{\text {dist }}(\chi)$ denote the proportion of distinct zeros of $L(s, \chi)$ in the critical strip. Combinatorial arguments as in [13] yield, for $m \geq 0$,

$$
\alpha_{\geq m+2}(\chi) \leq \frac{m+2}{2}\left(1-\alpha_{\mathrm{s}}^{(m)}(\chi)\right)
$$


and for $M \geq 0$

$$
\alpha_{\text {dist }}(\chi) \geq 2^{-M}\left(\alpha_{\mathrm{s}}^{(M)}(\chi)+\sum_{m=0}^{M-1} 2^{M-1-m} \alpha_{\mathrm{s}}^{(m)}(\chi)\right) .
$$

Therefore for example,

$$
\alpha_{\geq 2}(\chi)<0.643731, \quad \alpha_{\geq 3}(\chi)<0.318324, \quad \ldots, \quad \alpha_{\geq 8}(\chi)<0.039072
$$

and

$$
\alpha_{\text {dist }}(\chi)>0.613470 \text {. }
$$

Similar but weaker bounds are known for Dirichlet series associated with holomorphic cusp forms ([13]), and, of course, better results may be obtained by using the better bounds known for $\zeta(s)$.

The reason for the difference between our results and these results about the Riemann $\zeta$-function or Dirichlet series associated with holomorphic cusp forms lies in the length of the mollifier $B_{y}\left(s, P_{j}, \chi\right)$ in Theorem 1. Concerning $\zeta(s)$, a length of $y=T^{\theta}$ with $\theta=4 / 7-\varepsilon$ could be used in [6] while we are restricted to $\theta=1 / 2-\varepsilon$. In [13], on the other hand, $\theta$ had to be bounded by $1 / 6$.

The method we use to prove our theorem is similar to that used in [8] or [6]. In the latter article the work of Deshouillers and Iwaniec on averages of Kloosterman sums ([10], [11]) allowed the choice of the longer mollifier in the case of the Riemann $\zeta$-function. Because of characters appearing in our formulas we have to use other arguments to bound our main error term. We do this by using large sieve estimates. This idea originally appeared in the context of $\zeta(s)$ in [7], but here we have to deal with a more complicated situation. The disadvantage of our method is that we have to work with a shorter mollifier.

The above corollaries may be proven by a straightforward modification of the method used e.g. in [6] using our Theorem 1 instead of Theorem 4 in [6]. It thus remains for us to show Theorem 1.

3. Proof of Theorem 1-the first part. The first steps of the proof are only outlined because similar calculations were published elsewhere (e.g. [8], [6]). All the details may be found in [3]. With the notations mentioned in Section 2, let

$$
M:=\frac{1}{i} \int_{c+i}^{c+i T} L(s+\alpha, \chi) B_{y}\left(s, P_{1}, \chi\right) L(1-s-\beta, \bar{\chi}) B_{y}\left(1-s, P_{2}, \bar{\chi}\right) d s
$$

where $\alpha=A / \mathcal{L}$ and $\beta=B / \mathcal{L}$. Using well known estimates, we can move 
the line of integration to $\sigma=c^{\prime}:=1+\varepsilon, \varepsilon>0$ fixed, and obtain

$$
\begin{aligned}
M= & \frac{1}{i} \int_{c^{\prime}+i}^{c^{\prime}+i T} L(s+\alpha, \chi) L(1-s-\beta, \bar{\chi}) B_{y}\left(s, P_{1}, \chi\right) B_{y}\left(1-s, P_{2}, \bar{\chi}\right) d s \\
& +O\left(q T^{1 / 3} y \log ^{6} q T \log ^{2} y\right) .
\end{aligned}
$$

Next, we use the functional equation in the form

$$
L(s, \chi)=h(s, \chi) L(1-s, \bar{\chi}),
$$

where $\chi$ is a primitive Dirichlet character, and

$$
h(s, \chi):=\frac{\varepsilon_{\chi} 2^{s-1} \pi^{s} q^{1 / 2-s}}{\cos \frac{\pi(s-a)}{2} \cdot \Gamma(s)} .
$$

Lemma 1. Let $c>1 / 2$ be fixed, $T$ sufficiently large, $r>0, \beta=B / \mathcal{L}$ where $|B| \leq \mathcal{A}, h(s, \chi)$ as above,

$$
E_{c}(r, T)=T^{c-1 / 2}+\frac{T^{c+1 / 2}}{|T-r|+T^{1 / 2}} \quad \text { and } \quad \tau(\chi)=\sum_{n=1}^{q} \chi(n) e\left(\frac{n}{q}\right) .
$$

If $r \leq(q T) /(2 \pi)$ then

$$
\begin{aligned}
& \int_{c+i}^{c+i T} h(1-s-\beta, \chi) r^{-s} d s \\
& \quad=2 \pi i \tau(\chi) e^{B} q^{-1} e\left(-\frac{r}{q}\right)+O\left(r^{-1} q^{1 / 2}+r^{-c} q^{c-1 / 2} E_{c}\left(\frac{2 \pi r}{q}, T\right)\right)
\end{aligned}
$$

and if $r>(q T) /(2 \pi)$ then

$$
\int_{c+i}^{c+i T} h(1-s-\beta, \chi) r^{-s} d s=O\left(r^{-c} q^{c-1 / 2} E_{c}\left(\frac{2 \pi}{q}, T\right)\right) .
$$

The implicit constants in the error terms are independent of $q, r$ and $B$, but may depend on $\mathcal{A}$.

This lemma follows from Stirling's formula using the saddle point method (cf. e.g. [19], [15], [3]).

Lemma 2. Let $E_{c}(r, T)$ be defined as in Lemma $1, \alpha=A / \mathcal{L}, \beta=B / \mathcal{L}$ and $\eta>0$ arbitrary, but fixed. Then

$$
\begin{aligned}
\sum_{h, k \leq y} \frac{k^{\eta}}{h^{1+\eta}} \sum_{n=1}^{\infty} \sum_{m=1}^{\infty} n^{-1-\eta-\alpha} m^{-1-\eta-\beta} E_{1+\eta} & \left(\frac{2 \pi n m h}{q k}, T\right) \\
& \ll T^{1 / 2+\eta} y^{1+\eta} \log T \cdot \log y .
\end{aligned}
$$

Similar estimates have been proven e.g. in [22], [23] or [19]. 
Using the functional equation and Lemmas 2 and 3, we obtain

$$
M=2 \pi \tau(\bar{\chi}) e^{B} q^{-1} \mathcal{N}+O\left(q^{1+\varepsilon} T^{1 / 2+\varepsilon} y^{1+\varepsilon}\right),
$$

where $\tau(\chi)$ is the Gaussian sum, defined in Lemma 1, and

$$
\begin{aligned}
\mathcal{N}= & \sum_{h, k \leq y} \frac{\chi(h) \bar{\chi}(k) b\left(h, P_{1}\right) b\left(k, P_{2}\right)}{k} \\
& \times \sum_{\substack{n, m \\
n m \leq(q T k) /(2 \pi h)}} \frac{\chi(n) \chi(m)}{n^{\alpha} m^{\beta}} e\left(-\frac{n m h}{q k}\right),
\end{aligned}
$$

and where $b\left(n, P_{j}\right):=\mu(n) P_{j}(1-\log n / \log y)$ denotes the $n$th coefficient of our mollifier $B_{y}\left(s, P_{j}, \chi\right)$.

Next, we need Perron's formula:

LEMma 3. Let $\sum_{n, m} a(n, m) /\left(n^{s} m^{s}\right)$ be absolutely convergent for $\sigma>1$ and let $|a(n, m)| \ll \Phi(n m)$ with some function $\Phi(N)$ that is strictly increasing for large $N$. Let, for some $k>0$,

$$
\sum_{n, m} \frac{|a(n, m)|}{n^{\sigma} m^{\sigma}} \ll \frac{1}{(\sigma-1)^{k}} \quad \text { as } \sigma \rightarrow 1^{+} .
$$

Then, for $1<c<2, x \geq 1$ and any $\tau \geq 1$, we have

$$
\begin{aligned}
\sum_{\substack{n, m \\
n m \leq \tau}} a(n, m)= & \frac{1}{2 \pi i} \int_{c-i x}^{c+i x} \sum_{n, m} \frac{a(n, m)}{n^{s} m^{s}} \cdot \frac{\tau^{s}}{s} d s \\
& +O\left(\frac{\tau^{c}}{x(c-1)^{k}}+\frac{\Phi(2 \tau) \tau \log 2 \tau}{x}+\Phi(2 \tau)\right) .
\end{aligned}
$$

This can be shown analogously to [21], Satz A.3.1.

Let $c=1+\eta, \eta>0$ be fixed, and $\tau=(q T k) /(2 \pi h)$. An application of Lemma 3 yields

$$
\begin{aligned}
\sum_{\substack{n, m \\
n m \leq \tau}} \frac{\chi(n) \bar{\chi}(m)}{n^{\alpha} m^{\beta}} e\left(-\frac{n m h}{q k}\right) & \\
= & \frac{1}{2 \pi i} \int_{c-i x}^{c+i x} \frac{\tau^{s}}{s} \sum_{n=1}^{\infty} \sum_{m=1}^{\infty} \frac{\chi(n) \chi(m)}{n^{s+\alpha} m^{s+\beta}} e\left(-\frac{n m h}{q k}\right) d s \\
& +O\left(\frac{\tau^{1+\eta}}{x \eta^{2}}+\frac{2^{2 \mathcal{A} / \mathcal{L}} \tau^{1+2 \mathcal{A} / \mathcal{L}} \log 2 \tau}{x}+(2 \tau)^{2 \mathcal{A} / \mathcal{L}}\right) .
\end{aligned}
$$


From this and (1) we deduce that

$$
\begin{aligned}
\mathcal{N}= & \frac{1}{2 \pi i} \sum_{h, k \leq y} \frac{\chi(h) \bar{\chi}(k) b\left(h, P_{1}\right) b\left(k, P_{2}\right)}{k} \\
& \times \int_{c-i x}^{c+i x} \frac{\tau^{s}}{s} \sum_{n=1}^{\infty} \sum_{m=1}^{\infty} \frac{\chi(n) \chi(m)}{n^{s+\alpha} m^{s+\beta}} e\left(-\frac{n m h}{q k}\right) d s+\mathcal{F}_{1}(x),
\end{aligned}
$$

where

$$
\mathcal{F}_{1}(x) \ll x^{-1} y^{1+\eta}\left(\frac{q T}{2 \pi}\right)^{1+\eta}+y \log y .
$$

We use Estermann's method ([12]) to split our last expression for $\mathcal{N}$ into a main term and an error term. Let $\zeta(s, x), 0<x \leq 1$, denote the Hurwitz $\zeta$-function, and let $H=h /(h, k)$ and $K=k /(h, k)$. For the two innermost sums in (2), one obtains

$$
\sum_{\substack{n=1 \\ m=1}}^{\infty} \frac{\chi(n) \chi(m)}{n^{s+\alpha} m^{s+\beta}} e\left(-\frac{n m h}{q k}\right)=(q K)^{-2 s-\alpha-\beta} D(s),
$$

where

$$
D(s):=\sum_{1 \leq \nu, \mu \leq q K} \chi(\nu) \chi(\mu) e\left(-\frac{\nu \mu h}{q k}\right) \zeta\left(s+\alpha, \frac{\nu}{q K}\right) \zeta\left(s+\beta, \frac{\mu}{q K}\right) .
$$

This representation is valid for all $\sigma>0$. Because $\zeta(s, x)-\zeta(s)$ is regular for $0<x \leq 1$ in the whole complex plane, the same is true for

$$
\begin{aligned}
D^{*}(s):= & \sum_{1 \leq \nu, \mu \leq q K} \chi(\nu) \chi(\mu) e\left(-\frac{\nu \mu H}{q K}\right) \\
& \times\left(\zeta\left(s+\alpha, \frac{\nu}{q K}\right)-\zeta(s+\alpha)\right)\left(\zeta\left(s+\beta, \frac{\mu}{q K}\right)-\zeta(s+\beta)\right) .
\end{aligned}
$$

Using

$$
\begin{aligned}
\sum_{1 \leq \nu \leq q K} \chi(\nu) e\left(-\frac{\nu \mu}{q K}\right) & =\sum_{1 \leq j \leq q} \chi(j) e\left(-\frac{j \mu H}{q K}\right) \sum_{0 \leq r \leq K-1} e\left(-\frac{r \mu H}{K}\right), \\
\sum_{j=1}^{q} \chi(j) e\left(-\frac{n j}{q}\right) & =\bar{\chi}(-n) \tau(\chi),
\end{aligned}
$$

and

$$
L(s, \chi)=\frac{1}{q} \sum_{j=1}^{q} \chi(j) \zeta\left(s, \frac{j}{q}\right) \quad \text { for } \sigma>0,0<x \leq 1,
$$


we get

$$
\begin{aligned}
\sum_{1 \leq \mu \leq q K} \chi(\mu) \zeta\left(s+\beta, \frac{\mu}{q K}\right) & \sum_{1 \leq \nu \leq q K} \chi(\nu) e\left(-\frac{\nu \mu H}{q K}\right) \\
& =K \tau(\chi) \chi(K) \bar{\chi}(-H) q^{s+\beta} L\left(s+\beta, \chi_{0}\right),
\end{aligned}
$$

where $\chi_{0}$ denotes the principal character modulo $q$. In an analogous manner we get

$$
\sum_{1 \leq \nu \leq q K} \chi(\nu) \sum_{1 \leq \mu \leq q K} \chi(\mu) e\left(-\frac{\nu \mu H}{q K}\right)=K \tau(\chi) \chi(K) \bar{\chi}(-H) \varphi(q)
$$

and thus $D^{*}(s)=D(s)-E_{1}(s)-E_{2}(s)+E_{3}(s)$, where

$$
\begin{aligned}
& E_{1}(s)=K q^{s+\beta} \tau(\chi) \chi(K) \bar{\chi}(-H) L\left(s+\beta, \chi_{0}\right) \zeta(s+\alpha), \\
& E_{2}(s)=K q^{s+\alpha} \tau(\chi) \chi(K) \bar{\chi}(-H) L\left(s+\alpha, \chi_{0}\right) \zeta(s+\beta), \\
& E_{3}(s)=K \varphi(q) \tau(\chi) \chi(K) \bar{\chi}(-H) \zeta(s+\alpha) \zeta(s+\beta) .
\end{aligned}
$$

If $R_{c}(x)$ denotes the closed rectangle with vertices at $c \pm i x$ and $1 / 2 \pm i x$ and if $\Gamma_{c}(x)$ denotes the path along the upper, left and lower part of $R_{c}(x)$, using (2) and (4), we obtain

(5) $\mathcal{N}=\frac{1}{2 \pi i} \sum_{h, k \leq y} \frac{\chi(h) \bar{\chi}(k) b\left(h, P_{1}\right) b\left(k, P_{2}\right)}{k} \int_{R_{c}(x)} \frac{\tau^{s}\left(E_{1}+E_{2}-E_{3}\right)(s)}{s(q K)^{2 s+\alpha+\beta}} d s$ $+\mathcal{F}_{1}(x)-\mathcal{F}_{2}(x)$,

where $\mathcal{F}_{1}(x)$ has been defined above, and where

$$
\mathcal{F}_{2}(x)=\frac{1}{2 \pi i} \sum_{h, k \leq y} \frac{\chi(h) \bar{\chi}(k) b\left(h, P_{1}\right) b\left(k, P_{2}\right)}{k} \int_{\Gamma_{c}(x)} \frac{\tau^{s} D(s)}{s(q K)^{-2 s-\alpha-\beta}} d s .
$$

Because $A \neq B$ (which is equivalent to $\alpha \neq \beta$ ), the residues at $1-\alpha$ and $1-\beta$ of the integral in (5) are given by

$$
\begin{aligned}
K^{-1+\alpha-\beta} q^{-1} & \tau(\chi) \bar{\chi}(-H) \chi(K) L\left(1-\alpha+\beta, \chi_{0}\right) \frac{\tau^{1-\alpha}}{1-\alpha} \\
& +K^{-1-\alpha+\beta} q^{-1} \tau(\chi) \bar{\chi}(-H) \chi(K) L\left(1+\alpha-\beta, \chi_{0}\right) \frac{\tau^{1-\beta}}{1-\beta}
\end{aligned}
$$

Hence

$$
\begin{aligned}
M= & T \frac{e^{B-A}}{1-\alpha} L\left(1-\alpha+\beta, \chi_{0}\right) S\left(-\alpha, \beta, \chi_{0}\right) \\
& +T \frac{1}{1-\beta} L\left(1+\alpha-\beta, \chi_{0}\right) S\left(-\beta, \alpha, \chi_{0}\right) \\
& +O\left(q^{-1 / 2}\left(\left|\mathcal{F}_{1}(x)\right|+\left|\mathcal{F}_{2}(x)\right|\right)+q^{1+\varepsilon} T^{1 / 2+\varepsilon} y^{1+\varepsilon}\right),
\end{aligned}
$$


where

$$
S\left(\alpha, \beta, \chi_{0}\right):=\sum_{h, k \leq y} \frac{b\left(h, P_{1}\right) b\left(k, P_{2}\right)}{h^{1+\alpha} k^{1+\beta}} \chi_{0}\left(\frac{h k}{(h, k)}\right)(h, k)^{1+\alpha+\beta} .
$$

4. Proof of Theorem 1-the main term. In order to get the main term of the assertion of Theorem 1 , we have to examine the sum $S\left(\alpha, \beta, \chi_{0}\right)$. Let

$$
F(d, s, \chi)=\prod_{p \mid d}\left(1-\frac{\chi(p)}{p^{s}}\right)=\sum_{e \mid d} \mu(e) \frac{\chi(e)}{e^{s}} .
$$

As defined above, $b(n, P)=\mu(n) P(1-\log n / \log y)$, with a polynomial $P$ satisfying $P(0)=0$. The Möbius inversion formula gives

$$
\begin{aligned}
S\left(\alpha, \beta, \chi_{0}\right)= & \sum_{d \leq y} \frac{F\left(d, 1+\alpha+\beta, \chi_{0}\right) \chi_{0}(d)}{d} \\
& \times \sum_{h \leq y / d} \frac{b\left(h d, P_{1}\right) \chi_{0}(h)}{h^{1+\alpha}} \sum_{k \leq y / d} \frac{b\left(k d, P_{2}\right) \chi_{0}(k)}{h^{1+\beta}} .
\end{aligned}
$$

Defining

$$
G_{P}\left(d, z, \chi_{0}\right):=\sum_{\substack{n \leq y / d \\(n, d)=1}} \frac{\mu(n)}{n^{z}} \chi_{0}(n) P\left(1-\frac{\log n d}{\log y}\right)
$$

we get

$$
\begin{aligned}
S\left(\alpha, \beta, \chi_{0}\right)= & \sum_{d \leq y} \frac{\mu(d)}{d} \chi_{0}(d) F\left(d, 1+\alpha+\beta, \chi_{0}\right) \\
& \times G_{P_{1}}\left(d, 1+\alpha, \chi_{0}\right) G_{P_{2}}\left(d, 1+\beta, \chi_{0}\right) .
\end{aligned}
$$

To obtain an asymptotic expression for this, we apply the following lemmas which may be proven along the same lines as the corresponding results of $[22],[19]$, or [5]:

Lemma 4. Let $G_{P}\left(d, z, \chi_{0}\right), \alpha, \chi_{0}$ be as above. If $d \leq y$ and $P$ is a polynomial satisfying $P(0)=0$, we have

$$
\begin{aligned}
G_{P}(d, 1+ & \left.\alpha, \chi_{0}\right) \\
= & \frac{q^{1+\alpha}}{\varphi(q)} F\left(d, 1+\alpha, \chi_{0}\right)^{-1}\left(\alpha P\left(\frac{\log y / d}{\log y}\right)+\log ^{-1} y P^{\prime}\left(\frac{\log y / d}{\log y}\right)\right) \\
& +O\left(F_{1}\left(d, 1-\delta, \chi_{0}\right)(\log \log y)^{3}\left(q \log ^{-2} y+\left(\frac{y}{d}\right)^{-\delta} \log ^{-1} y\right)\right),
\end{aligned}
$$

where $F_{1}(d, s, \chi)=\prod_{p \mid d}\left(1+\chi(p) p^{-s}\right)$. 
Lemma 5. Let $f(p)=1+O\left(p^{-c}\right), c>0$ and $f(d)=\prod_{p \mid d} f(p)$. If $\chi_{0}$ denotes the principal character modulo $q \geq 1$, we have, for $\nu \geq 0$,

$\sum_{d \leq y} \frac{\mu^{2}(d)}{d} f(d) \log ^{\nu} \frac{y}{d}=\mathcal{P}_{f} \cdot \frac{\varphi(q)}{q} \cdot L\left(2, \chi_{0}\right)^{-1} \cdot(\nu+1)^{-1} \log ^{\nu+1} y+O_{\nu}\left(\log ^{\nu} y\right)$

where

$$
\mathcal{P}_{f}:=\prod_{p}\left(1+\frac{f(p)-\chi_{0}(p)}{\chi_{0}(p)-p}\right)
$$

is absolutely convergent. In the special case

$$
f(p)=\chi_{0}(p) \frac{1-\chi_{0}(p) p^{-1-\alpha-\beta}}{\left(1-\chi_{0}(p) p^{-1-\alpha}\right)\left(1-\chi_{0}(p) p^{-1-\beta}\right)},
$$

we have

$$
\mathcal{P}_{f} \cdot L\left(2, \chi_{0}\right)^{-1}=1+O\left(\mathcal{L}^{-1}\right) \quad \text { if } \alpha, \beta \ll \mathcal{L}^{-1}
$$

Lemma 6. Let $\delta \geq 0, \delta^{\prime} \geq 0$ and $\delta+\delta^{\prime} \leq c<1$. Further let $F_{1}(d, s, \chi)=$ $\prod_{p \mid d}\left(1+\chi(p) / p^{s}\right)$ as above. Then for $r=1,2, \ldots$, we have

$$
\sum_{d \leq y} \frac{\mu^{2}(d)}{d^{1-\delta}} F_{1}\left(d, 1-\delta^{\prime}, \chi_{0}\right)^{r}= \begin{cases}O_{c, r}(\log y) & \text { if } \delta=0 \\ O_{c, r}\left(y^{\delta} / \delta\right) & \text { if } \delta>0\end{cases}
$$

Using Lemma 4 and our last representation (6) of $S\left(\alpha, \beta, \chi_{0}\right)$, we get terms of the form

$$
\sum_{d \leq y} \frac{\mu^{2}(d)}{d} f(d) P\left(1-\frac{\log d}{\log y}\right)=\sum_{\nu \geq 0} a_{\nu} \log ^{-\nu} y \sum_{d \leq y} \frac{\mu^{2}(d)}{d} f(d) \log ^{\nu} \frac{y}{d} .
$$

By Lemma 5 , this equals $\frac{\varphi(q)}{q} \log y \int_{0}^{1} P(t) d t+O_{P}(1)$. Using Lemma 6 to bound the error terms, we get

$$
\begin{aligned}
S\left(\alpha, \beta, \chi_{0}\right)= & \frac{q^{1+\alpha+\beta}}{\varphi(q)} \cdot \frac{\theta}{\mathcal{L}} \int_{0}^{1}\left(A P_{1}(t)+\frac{1}{\theta} P_{1}^{\prime}(t)\right)\left(B P_{2}(t)+\frac{1}{\theta} P_{2}^{\prime}(t)\right) d t \\
& +O\left(\frac{q^{2} \log q}{\varphi(q)}(\log \log y)^{7} \log ^{-2} y\right) .
\end{aligned}
$$

Since $P_{1}(0)=P_{2}(0)=0$ the formula $\int_{0}^{1}\left(P_{1}^{\prime} P_{2}+P_{1} P_{2}^{\prime}\right)(t) d t=P_{1}(1) P_{2}(1)$ holds, and we obtain

$$
\begin{aligned}
S\left(-\alpha, \beta, \chi_{0}\right)- & S\left(-\beta, \alpha, \chi_{0}\right) \\
& =\frac{q}{\varphi(q)} \cdot \frac{B-A}{\mathcal{L}} \cdot P_{1}(1) P_{2}(1)+O\left(\frac{q \log q}{\varphi(q)} \mathcal{L}^{-2} \log ^{7} \mathcal{L}\right) .
\end{aligned}
$$


Collecting our results, we obtain the following formula for the mean value in question:

$$
\begin{array}{r}
M=T\left(\frac{e^{B-A}-1}{B-A} \cdot \theta \int_{0}^{1}\left(-A P_{1}(t)+\frac{1}{\theta} P_{1}^{\prime}(t)\right)\left(B P_{2}(t)+\frac{1}{\theta} P_{2}^{\prime}(t)\right) d t\right. \\
\left.+P_{1}(1) P_{2}(1)\right) \\
+O\left(T \mathcal{L}^{-1} \log q \cdot \log ^{7} \mathcal{L}+q^{1 / 2+\varepsilon} T^{1 / 2+\varepsilon} y^{1+\varepsilon}+q^{-1 / 2}\left(\left|\mathcal{F}_{1}(x)\right|+\left|\mathcal{F}_{2}(x)\right|\right)\right) .
\end{array}
$$

5. Proof of Theorem 1 - the error term. It remains to bound the error terms. $\mathcal{F}_{1}(x)$ has been bounded in $(3)$. We shall see that it will be convenient to choose $x=T^{n}$ with some large absolute constant $n$. This will yield $\mathcal{F}_{1}\left(T^{n}\right) \sim y \log y=T^{\theta} \cdot \theta \log T$ if $T \rightarrow \infty$. The choice of $x$ depends on $\mathcal{F}_{2}$, and the estimation of this error term is the most critical step in the proof of Theorem 1.

Using the notation of Section 3, we easily see that, with the path $\Gamma_{1+\eta}(x)$ defined before equation (5),

$$
\mathcal{F}_{2}(x)=\int_{\Gamma_{1+\eta}(x)} \sum_{k \leq y} \frac{\bar{\chi}(k) b\left(k, P_{2}\right)}{k^{1-s}} \cdot \mathcal{H}(s, k)\left(\frac{q T}{2 \pi}\right)^{s} \frac{d s}{s},
$$

where

$$
\begin{aligned}
\mathcal{H}(s, k) & =\sum_{h \leq y} \frac{b\left(h, P_{1}\right)}{h^{s}} \sum_{n=1}^{\infty} \sum_{m=1}^{\infty} \frac{\chi(h n m)}{n^{s+\alpha} m^{s+\beta}} e\left(-\frac{n m h}{q k}\right) \\
& =\sum_{j=1}^{\infty} j^{-s} a(j) e\left(-\frac{j}{q k}\right) .
\end{aligned}
$$

Here, the coefficients are given by

$$
a(j)=\sum \frac{b\left(h, P_{1}\right) \chi(h n m)}{n^{\alpha} m^{\beta}},
$$

where the summation is over all $n, m, h$ satisfying $h<y$ and $n m h=j$. Put $J=j /(j, q k), Q=q k /(j, q k)$, and let $\sum_{\chi \bmod q}$ denote a sum over all characters modulo $q$. Using the Möbius inversion formula and

$$
e\left(\frac{j}{q}\right)=\frac{1}{\varphi(q)} \sum_{\chi \bmod q} \tau(\bar{\chi}) \chi(j)
$$

we obtain

$$
e\left(-\frac{j}{q k}\right)=\sum_{d \mid(j, q k)} \sum_{e \mid d} \mu\left(\frac{d}{e}\right) \varphi\left(\frac{q k}{e}\right)^{-1} \sum_{\psi \bmod q k / e} \tau(\bar{\psi}) \psi\left(-\frac{j}{e}\right) .
$$


This implies

$$
\mathcal{H}(s, k)=\sum_{d \mid q k} \frac{1}{d^{s} \varphi(q k / d)} \sum_{\psi \bmod q k / d} \psi(-1) \tau(\bar{\psi}) A(s, d, \psi),
$$

where $A(s, d, \psi)$ denotes the Dirichlet series

$$
\begin{aligned}
A(s, d, \psi) & =\sum_{j=1}^{\infty} a(j d) \psi(j) j^{-s} \\
= & \sum_{d_{1} d_{2} d_{3}=d}\left(\sum_{n=1}^{\infty} \frac{\psi(n) \chi\left(n d_{1}\right)}{d_{1}^{\alpha} n^{s+\alpha}}\right)\left(\sum_{\substack{m=1 \\
\left(m, d_{1}\right)=1}}^{\infty} \frac{\psi(m) \chi\left(m d_{2}\right)}{d_{2}^{\beta} m^{s+\beta}}\right) \\
& \left.\times \sum_{\substack{h \leq y / d_{3} \\
\left(m, d_{1} d_{2}\right)=1}} \frac{\psi(h) \chi\left(h d_{3}\right) b\left(h d_{3}, P_{1}\right)}{h^{s}}\right) \\
= & \quad L(s+\alpha, \psi \chi) L(s+\beta, \psi \chi) \\
& \times \sum_{d_{1} d_{2} d_{3}=d} \frac{\chi\left(d_{1} d_{2}\right) F\left(d_{1}, s+\beta, \psi \chi\right)}{d_{1}^{\alpha} d_{2}^{\beta}} \sum_{\substack{h \leq y / d_{3} \\
\left(h, d_{1} d_{2}\right)=1}} \frac{b\left(h d_{2}, P_{1}\right) \chi\left(h d_{3}\right) \psi(h)}{h^{s}} .
\end{aligned}
$$

The infinite series is convergent for $\sigma>1$, therefore, by analytic continuation, the last expression is valid for $\sigma \leq 1$ as well.

Let $\tau_{n}=1 * \ldots * 1$ denote the $n$th divisor function. Because of $F(d, s, \chi) \ll$ $\tau_{2}(d)$ and $\tau_{n}(d) \tau_{m}(d) \leq \tau_{n m}(d)$,

$$
A(s, d, \psi) \ll \tau_{6}(d)|L(s+\alpha, \psi \chi) L(s+\beta, \psi \chi) \Psi(s, \psi)|
$$

where $\Psi(s, \psi)=\sum_{h \leq y} \psi(h) c(h) h^{-s}$ represents a Dirichlet series with coefficients $c(h) \ll 1$.

First we bound $\mathcal{F}_{2}(x)$ on the horizontal parts of $\Gamma_{1+\eta}(x)$. Using $L(\sigma+i x, \chi) \ll x^{1 / 6} q^{1 / 2} \log ^{3} q x$ for $x \geq 2, \sigma \geq 1 / 2$ and all characters $\chi \bmod q$ ([18]) and $\Psi(\sigma+i x, \psi) \ll \sum_{h \leq y} h^{-\sigma} \ll y^{1-\sigma} \log y$ for $\sigma>0$, we find that for any $\varepsilon>0$,

$$
A(\sigma+i x, d, \psi) \ll \tau_{6}(d)[q, q k / d]^{1+\varepsilon} x^{1 / 3+\varepsilon} y^{1-\sigma} \log y .
$$

Here, and in the sequel, $[n, m]$ denotes the least common multiple of $n$ and $m$. Hence

$$
\begin{aligned}
\mathcal{H}(\sigma+i x, k) & \ll x^{1 / 3+\varepsilon} y^{1-\sigma} \log y \sum_{d \mid q k} \frac{\tau_{6}(d)}{d^{\sigma} \varphi(q k / d)}[q, q k / d]^{1+\varepsilon} \sum_{\psi \bmod q k / d}|\tau(\bar{\psi})| \\
& \ll x^{1 / 3+\varepsilon}(q k)^{3 / 2+\varepsilon} \tau_{7}(q) \tau_{7}(k) y^{1-\sigma} \log y,
\end{aligned}
$$

by (9) and the estimate for Gaussian sums $|\tau(\bar{\psi})| \leq \sqrt{q k / d}$ (cf. e.g. [9], §9). 
So, because of (8) the contribution of the horizontal parts $s=\sigma \pm i x$ of $\Gamma_{1+\eta}(x)$ to the error term $\mathcal{F}_{2}(x)$ is

$$
\begin{aligned}
\int_{1 / 2}^{1+\eta} \sum_{k \leq y} \frac{\bar{\chi}(k) b\left(k, P_{2}\right)}{k^{1-s}} & \mathcal{H}(s, k)\left(\frac{q T}{2 \pi}\right)^{s} \frac{d \sigma}{s} \\
\ll & q^{3 / 2+\varepsilon} \tau_{7}(q) x^{1 / 3+\varepsilon} y \log y \\
& \times \int_{1 / 2}^{1+\eta} y^{-\sigma}\left(\frac{q T}{2 \pi}\right)^{\sigma} \sum_{k \leq y} k^{1 / 2+\sigma+\varepsilon} \tau_{7}(k) \frac{d \sigma}{\left(\sigma^{2}+x^{2}\right)^{1 / 2}} \\
\ll & q^{5 / 2+\eta+\varepsilon} \tau_{7}(q) x^{-2 / 3+\varepsilon} T^{1+\eta} y^{3-\eta+\varepsilon} \log ^{q} y=: \mathcal{F}_{21}(x) .
\end{aligned}
$$

If we choose $x=T^{n}$ with a large absolute constant $n$, this part of the error term will become sufficiently small.

Next we bound $\mathcal{F}_{2}(x)$ on the vertical part of $\Gamma_{1+\eta}(x)$, where $\sigma=1 / 2$ and $|t| \leq x$. If $\chi$ is a character $\bmod q$, let $\chi^{*}$ denote the primitive character modulo the conductor of $\chi$ satisfying $\chi(n)=\chi_{0}(n) \chi^{*}(n)$ for all $n$, where $\chi_{0}$ denotes the principal character mod $q$ (cf. e.g. [1], §8.9).

Using this notation and some well known estimates (e.g. [4]), we have $L(s, \psi \chi) \ll[q, \kappa]^{\varepsilon}\left|L\left(s,(\psi \chi)^{*}\right)\right|$ for $\sigma>0, \varepsilon>0$ and any characters $\psi \bmod \kappa$ and $\chi \bmod q$.

Applying this result, (9), and (10), we obtain

$$
\begin{aligned}
\sum_{k \leq y} \frac{\bar{\chi}(k) b\left(k, P_{2}\right)}{k^{1-s}} & \mathcal{H}(s, k) \\
\ll & \frac{1}{q^{1 / 2-\varepsilon}} \sum_{k \leq y} \frac{1}{k^{1-\varepsilon}} \sum_{d \mid q k} \frac{\tau_{6}(d)}{d^{\varepsilon}}\left[q, \frac{q k}{d}\right]^{\varepsilon} \\
& \times \sum_{\psi \bmod q k / d}\left|L\left(s+\alpha,(\psi \chi)^{*}\right) L\left(s+\beta,(\psi \chi)^{*}\right) \Psi(s, \psi)\right|,
\end{aligned}
$$

and thus the contribution of the vertical part of $\Gamma_{1+\eta}(x)$ to $\mathcal{F}_{2}(x)$ is

$$
\begin{aligned}
\int_{1 / 2-i x}^{1 / 2+i x} \sum_{k \leq y} & \frac{\bar{\chi}(k) b\left(k, P_{2}\right)}{k^{1-s}} \mathcal{H}(s, k)\left(\frac{q T}{2 \pi}\right)^{s} \frac{d s}{s} \\
\ll & q^{\varepsilon} T^{1 / 2} \sum_{k \leq y} k^{-1+\varepsilon} \sum_{d \mid q k} \frac{\tau_{6}(d)}{d^{\varepsilon}}\left[q, \frac{q k}{d}\right]^{\varepsilon} \\
& \times \sum_{\psi \bmod q k / d} \int_{-x}^{x}\left|L\left(s+\alpha,(\psi \chi)^{*}\right) L\left(s+\beta,(\psi \chi)^{*}\right) \Psi(s, \psi)\right| \frac{d t}{|1 / 2+i t|} \\
= & : \mathcal{F}_{22}(x) .
\end{aligned}
$$


Using Hölder's inequality, we can split the innermost sum into three factors:

$$
\begin{aligned}
\sum_{\psi \bmod q k / d} \int_{-x}^{x} \mid L(s+ & \left.\alpha,(\psi \chi)^{*}\right) L\left(s+\beta,(\psi \chi)^{*}\right) \Psi(s, \psi) \mid \frac{d t}{|1 / 2+i t|} \\
\leq & \left(\sum_{\psi \bmod q k / d} \int_{-x}^{x}\left|L\left(1 / 2+\alpha+i t,(\psi \chi)^{*}\right)\right|^{4} \frac{d t}{|1 / 2+i t|}\right)^{1 / 4} \\
& \times\left(\sum_{\psi \bmod q k / d} \int_{-x}^{x}\left|L\left(1 / 2+\beta+i t,(\psi \chi)^{*}\right)\right|^{4} \frac{d t}{|1 / 2+i t|}\right)^{1 / 4} \\
& \times\left(\sum_{\psi \bmod q k / d} \int_{-x}^{x}|\Psi(1 / 2+i t, \psi)|^{2} \frac{d t}{|1 / 2+i t|}\right)^{1 / 2} .
\end{aligned}
$$

In order to deal with these terms, we make use of the following two lemmas from the theory of the large sieve.

Lemma 7. If $x \geq 2$ and $|1 / 2-\sigma| \ll(\log q x)^{-1}$, then

$$
\sum_{\chi \bmod }^{*} \int_{-x}^{x}|L(s, \chi)|^{4} \frac{d t}{|1 / 2+i t|} \ll \varphi(q)\left(\log x \cdot \log ^{4} q x\right) .
$$

This consequence of a mean value theorem of Montgomery ([20], Thm. 10.1) may be obtained by partial summation. In the same manner, but using a result of Gallagher ([14], Thm. 2), we get

Lemma 8. Let $\chi$ denote a character mod $q$, and let

$$
S(t, \chi)=\sum_{n \leq y} a_{n} \chi(n) n^{-i t}
$$

with some arbitrary complex coefficients $a_{n}$. For $x \geq 2$,

$$
\sum_{\chi \bmod } \int_{-x}^{x}|S(t, \chi)|^{2} \frac{d t}{|1 / 2+i t|} \ll \sum_{n \leq y}(q \log x+n)\left|a_{n}\right|^{2} .
$$

Applying Lemma 7 and using $\alpha, \beta \ll \mathcal{L}$ and $x=T^{n}$ with some sufficiently large absolute constant $n$ (e.g. $n=10$ ), we obtain

$$
\sum_{\psi \bmod q k / d} \int_{-x}^{x}\left|L\left(1 / 2+\alpha+i t,(\psi \chi)^{*}\right)\right|^{4} \frac{d t}{|1 / 2+i t|} \ll[q, q k / d] \log ^{5}(x[q, q k / d]) .
$$

Analogously, applying Lemma 8 instead of Lemma 7, we obtain

$$
\begin{aligned}
\sum_{\psi \bmod } \int_{-x}^{x}|\Psi(1 / 2+i t, \psi)|^{2} \frac{d t}{|1 / 2+i t|} & \ll \sum_{\nu \leq y} \frac{(m \log x+\nu)|c(\nu)|^{2}}{\nu} \\
& \ll m \log x \cdot \log y+y,
\end{aligned}
$$


where we have used the representation $\Psi(s, \psi)=\sum_{\nu \leq y} \psi(\nu) c(\nu) \nu^{-s}$. Collecting our results, the error resulting from the vertical part of $\Gamma_{1+\eta}(x)$ is

$$
\begin{aligned}
\mathcal{F}_{22}(x) \ll & q^{\varepsilon} T^{1 / 2} \sum_{k \leq y} k^{-1+\varepsilon} \sum_{d \mid q k} \tau_{6}(d)\left(\frac{q k}{d}\right)^{\varepsilon} \\
& \times(q k)^{1 / 2} \log ^{5 / 2}(x q k)\left(y+\frac{q k}{d} \log x \cdot \log y\right)^{1 / 2} \\
& \ll q^{1+\varepsilon} T^{1 / 2+\varepsilon} y^{1+\varepsilon} .
\end{aligned}
$$

If we use $x=T^{n}, \mathcal{F}_{1}(x) \sim y \log y$ and $\mathcal{F}_{21}=o(1)$ as $T \rightarrow \infty$, the error term in (7) becomes

$$
\ll q T \mathcal{L}^{-1} \log q \cdot \log ^{7} \mathcal{L}+q^{1 / 2+\varepsilon} T^{1 / 2+\varepsilon} y^{1+\varepsilon} .
$$

Since $y=T^{\theta}$ with $\theta \leq \theta_{2}<1 / 2$ this completes the proof of Theorem 1 .

\section{References}

[1] T. M. Apostol, Introduction to Analytic Number Theory, Springer, 1986.

[2] P. J. Bauer, Über den Anteil der Nullstellen der Riemannschen Zeta-Funktion auf der kritischen Geraden, Diploma thesis, Frankfurt a.M., 1992. (Available at http://www.math.uni-frankfurt.de/ pbauer/diplom.ps.)

[3] -, Zur Verteilung der Nullstellen der Dirichletschen L-Reihen, Dissertation, Frankfurt a.M., 1997. (Available at http://www.math.uni-frankfurt.de/ ${ }^{\sim}$ pbauer/diss.ps.)

[4] D. A. Burgess, On character sums and L-series, Proc. London Math. Soc. 12 (1962), 193-206.

[5] J. B. Conrey, Zeros of derivatives of Riemann's Xi-function on the critical line, J. Number Theory 16 (1983), 49-74.

[6] - More than two fifths of the zeros of the Riemann zeta function are on the critical line, J. Reine Angew. Math. 399 (1989), 1-26.

[7] J. B. Conrey and A. Ghosh, A simpler proof of Levinson's theorem, Math. Proc. Cambridge Philos. Soc. 97 (1985), 385-395.

[8] J. B. Conrey, A. Ghosh and S. M. Gonek, Mean values of the Riemann zetafunction with application to the distribution of zeros, in: Number Theory, Trace Formulas and Discrete Groups, K. E. Aubert et al. (eds.), Academic Press, 1989, $185-199$.

[9] H. Davenport, Multiplicative Number Theory, 2nd ed., Springer, 1980.

[10] J.-M. Deshouillers and H. Iwaniec, Kloosterman sums and Fourier coefficients of cusp forms, Invent. Math. 70 (1982), 219-288.

[11] - - - Power mean-values for Dirichlet's polynomials and the Riemann zeta-function II, Acta Arith. 43 (1984), 305-312.

[12] T. Estermann, On the representation of a number as the sum of two products, Proc. London Math. Soc. (2) 31 (1930), 123-133.

[13] D. W. Farmer, Mean value of Dirichlet series associated with holomorphic cusp forms, J. Number Theory 49 (1994), 209-245.

[14] P. X. Gallagher, A large sieve density estimate near $\sigma=1$, Invent. Math. 11 (1970), 329-339. 
[15] S. M. Gonek, Mean values of the Riemann zeta-function and its derivatives, ibid. 75 (1984), 123-141.

[16] T. Hilano, On the distribution of zeros of Dirichlet's L-function on the line $\sigma=$ 1/2, Proc. Japan Acad. 52 (1976), 537-540.

[17] - On the distribution of zeros of Dirichlet's L-function on the line $\sigma=1 / 2(I I)$, Tokyo J. Math. 1 (1978), 285-304.

[18] G. Kolesnik, On the order of Dirichlet L-functions, Pacific J. Math. 82 (1979), 479-484.

[19] N. Levinson, More than one third of the zeros of Riemann's zeta-function are on $\sigma=1 / 2$, Adv. Math. 13 (1974), 383-436.

[20] H. L. Montgomery, Topics in Multiplicative Number Theory, Springer, 1971.

[21] K. Prachar, Primzahlverteilung, Springer, 1957.

[22] A. Selberg, On the zeros of Riemann's zeta-function, Skr. Norske VidenskapsAkad. Oslo, I, 10 (1942), 1-59.

[23] E. C. Titchmarsh, The Theory of the Riemann Zeta-Function, 2nd ed., Clarendon Press, 1988.

Fachbereich Mathematik

Johann Wolfgang Goethe-Universität

Robert-Mayer-Straße 6-10

60054 Frankfurt/Main, Germany

E-mail: pbauer@math.uni-frankfurt.de

Received on 25.1.1999

and in revised form on 15.11.1999 